\title{
Clinical Practice Recommendations on Genetic Testing of CYP2C9 and VKORC1 Variants in Warfarin Therapy
}

\author{
Kaitlyn Shaw, MSc, *† Ursula Amstutz, PhD, \& Richard B. Kim, MD, 9 Lawrence J. Lesko, PhD, \| \\ Jacques Turgeon, PhD,** Veronique Michaud, PhD, ** Soomi Hwang, †† Shinya Ito, MD, $\neq t$ \\ Colin Ross, PhD,* $\neq$ and Bruce C. Carleton, PharmD, * $\neq$ the CPNDS Clinical Recommendation Group
}

\begin{abstract}
Objective: To systematically review evidence on genetic variants influencing outcomes during warfarin therapy and provide practice recommendations addressing the key questions: (1) Should genetic testing be performed in patients with an indication for warfarin therapy to improve achievement of stable anticoagulation and reduce adverse effects? (2) Are there subgroups of patients who may benefit more from genetic testing compared with others? (3) How should patients with an indication for warfarin therapy be managed based on their genetic test results?
\end{abstract}

Methods: A systematic literature search was performed for VKORC1 and CYP2C9 and their association with warfarin therapy. Evidence was critically appraised, and clinical practice recommendations were developed based on expert group consensus.

Results: Testing of VKORC1 $(-1639 \mathrm{G}>\mathrm{A}), C Y P 2 C 9^{*} 2$, and $C Y P 2 C 9^{*} 3$ should be considered for all patients, including pediatric patients, within the first 2 weeks of therapy or after a bleeding event. Testing for $C Y P 2 C 9 * 5, * 6, * 8$, or $* 11$ and $C Y P 4 F 2$ (V433M) is currently not recommended. Testing should also be considered for all patients who are at increased risk of bleeding complications, who

Received for publication September 17, 2014; accepted January 19, 2015.

From the *Division of Translational Therapeutics, Department of Pediatrics, University of British Columbia; $†$ Pharmaceutical Outcomes Programme, British Columbia Children's Hospital; †Child and Family Research Institute, Vancouver, British Columbia, Canada; §Institute of Clinical Chemistry, Inselspital Bern University Hospital, University of Bern, Switzerland; qDivision of Clinical Pharmacology, Department of Medicine, Western University, London, Ontario, Canada; ||Department of Pharmaceutics, University of Florida, Orlando; **CRCHUM, Centre Hospitalier de l'Université de Montréal and Faculty of Pharmacy, Université de Montréal, Québec; $\dagger \dagger$ Faculty of Pharmaceutical Sciences, University of British Columbia, Vancouver; and țivision of Clinical Pharmacology and Toxicology, Department of Pediatrics, The Hospital for Sick Children, University of Toronto, Ontario, Canada.

Supported by a Canadian Institutes of Health Research (CIHR) Meetings, Planning and Dissemination Grant-Knowledge Translation Supplement, FRN 114403.

The authors declare no conflict of interest.

A full list of members of the CPNDS Clinical Recommendation Group is provided in the Acknowledgments.

Supplemental digital content is available for this article. Direct URL citations appear in the printed text and are provided in the HTML and PDF versions of this article on the journal's Web site (www.drug-monitoring.com).

Correspondence: Bruce C. Carleton, PharmD, Child and Family Research Institute, 950 West 28th Avenue, Vancouver, British Columbia, Canada V5Z 4H4 (e-mail: bcarleton@popi.ubc.ca).

Copyright $(C) 2015$ Wolters Kluwer Health, Inc. All rights reserved. consistently show out-of-range international normalized ratios, or suffer adverse events while receiving warfarin. Genotyping results should be interpreted using a pharmacogenetic dosing algorithm to estimate the required dose.

Significance: This review provides the latest update on genetic markers for warfarin therapy, clinical practice recommendations as a basis for informed decision making regarding the use of genotypeguided dosing in patients with an indication for warfarin therapy, and identifies knowledge gaps to guide future research.

Key Words: warfarin, VKORC1, CYP2C9, clinical practice recommendations, genetic testing

(Ther Drug Monit 2015;37:428-436)

\section{INTRODUCTION}

\section{Warfarin}

Warfarin is an oral anticoagulant used for the prevention and treatment of thromboembolic events. The list of indications for warfarin is extensive but includes atrial fibrillation, prosthetic heart valves, history of vascular thrombosis, or after orthopedic surgery. ${ }^{1}$ Warfarin is still widely considered the mainstay of anticoagulation therapy, with approximately 2 million people started on warfarin annually in the United States. ${ }^{2}$

Warfarin is administered as a racemic mixture of $\mathrm{R}-$ and S-enantiomers. S-warfarin is approximately 2-5 times more potent than R-warfarin and is almost exclusively metabolized by the cytochrome P450 2C9 (CYP2C9) enzyme in the liver. ${ }^{1,3}$ Warfarin acts as an anticoagulant by inhibiting the vitamin $\mathrm{K}$ epoxide reductase complex (VKORC1) and blocking regeneration of reduced vitamin $\mathrm{K}$ that is essential for the activation of specific coagulation factors. ${ }^{1,4,5}$

\section{Warfarin Dosing and Adverse Drug Reactions}

Clinical management and therapeutic monitoring of warfarin is achieved using the International Normalized Ratio (INR). A population-based initial warfarin dose is prescribed (eg, 5-10 mg/d), and subsequent dose changes are made to achieve an INR in the therapeutic range typically between 2 and 3. Use of warfarin is limited by a large interpatient variability in dose requirement, making both safe and effective therapeutic dosing of warfarin difficult to achieve. An INR below 2 increases the risk of thromboembolism, whereas an 
INR above 4 greatly increases the risk of bleeding. ${ }^{6,7}$ Achieving the target INR can take weeks and puts patients at increased risk of adverse drug reactions (ADRs) during the initiation period. ${ }^{8}$ Bleeding is the most common ADR associated with warfarin therapy, with the incidence of major bleeding ranging from $0 \%$ to $16 \%$ and the incidence of fatal bleeding reported as $0 \%-2.9 \%{ }^{9}$ Warfarin is the second most common drug implicated in emergency department visits and the most often cited cause of drug-related mortality. ${ }^{10,11}$

\section{Genetic Variants \\ CYP2C9}

CYP2C9 is a member of the cytochrome P450 superfamily of enzymes, which are responsible for the metabolism and elimination of many common prescription drugs. Approximately $5 \%-30 \%$ of the population carry variants of the CYP2C9 gene, which result in an enzyme with reduced or 0 activity. ${ }^{12}$ Patients carrying such variants have an impaired ability to metabolize warfarin, resulting in increased levels of the active S-warfarin metabolite and a decreased dose requirement to obtain therapeutic INR. ${ }^{13}$ In Europeans, the 2 most common variants that confer reduced enzyme activity are $C Y P 2 C 9^{*} 2$ and $C Y P 2 C 9^{*} 3$ (see Table 1, Supplemental Digital Content 1, http:/links. lww.com/TDM/A107). ${ }^{14}$ The frequencies of both variants are lower in African and Asian populations (Table 1). Additional CYP2C9 gene variants, such as CYP2C9*5, *6, *8, and * 11 , also result in reduced enzyme activity and are more common in African Americans. ${ }^{15-18}$

\section{VKORC1}

A specific variant in $V K O R C 1(-1639 \mathrm{G}>\mathrm{A})$ alters the rate at which the gene is transcribed and results in lower protein expression. Patients who carry the A variant require lower warfarin doses, whereas patients who carry 2 copies of the VKORC1-1639 G allele are more resistant to the anticoagulation effects of warfarin (see Table 2, Supplemental Digital Content 1, http://links.lww.com/TDM/A107). ${ }^{19}$ Therefore, this single-nucleotide polymorphism can be used to differentiate patients with high (AA), normal (AG), and low (GG) warfarin sensitivity. As with the CYP2C9 variants, the frequency of the $-1639 \mathrm{G}>\mathrm{A}$ variant differs among ethnic groups, occurring most frequently in Asians $(82 \%-96 \%)^{20}$ (Table 1). This finding helps explain historical interethnic differences in warfarin dose requirements, as Asians on average require a lower warfarin

TABLE 1. Allele Frequencies Across Ethnic Groups for CYP2C9 and VKORC1 ${ }^{20}$

\begin{tabular}{lccc}
\hline & \multicolumn{3}{c}{ Ethnic Group } \\
\cline { 2 - 4 } Allele & European, \% & African, \% & Asian, \% \\
\hline$C Y P 2 C 9^{*} 2$ & $8-15$ & $0-4$ & 0 \\
$C Y P 2 C 9^{*} 3$ & $6-8$ & $0-2$ & $2-5$ \\
$C Y P 2 C 9^{*} 5$ & 0 & $1-3$ & 0 \\
$C Y P 2 C 9^{*} 6$ & 0 & $0-2$ & 0 \\
$C Y P 2 C 9^{*} 8$ & 0 & $2-8$ & 0 \\
$C Y P 2 C 9^{*} 11$ & 0 & $1-5$ & 0 \\
VKORC1 $(-1639 \mathrm{G}>\mathrm{A})$ & $31-48$ & $3-15$ & $82-96$ \\
\hline
\end{tabular}

dose compared with Caucasians and Africans. ${ }^{19}$ Several rare point mutations in $V K O R C 1$ have also been associated with warfarin resistance. ${ }^{21-24}$ These variants occur in the coding region of the gene and lead to changes in the VKORC1 protein sequence, potentially affecting the warfarin site of action. ${ }^{22}$ However, the function of VKORC1 in extreme warfarin resistance is still poorly understood.

\section{CYP4F2}

CYP4F2 is a vitamin $\mathrm{K}$ oxidase that catalyzes the metabolism of vitamin $\mathrm{K}$ to hydroxyvitamin $\mathrm{K} 1$ and functions as a counterpart to VKORC1 to prevent accumulation of vitamin $\mathrm{K}$. A functional polymorphism in the $C Y P 4 F 2$ gene (V433M) encodes a protein with decreased activity, resulting in increased levels of vitamin $\mathrm{K} .{ }^{25} \mathrm{An}$ increase in the amount of vitamin $\mathrm{K}$ available for the activation of vitamin $\mathrm{K}$-dependent clotting factors results in a higher warfarin dose requirement in patients carrying this polymorphism. ${ }^{25}$

\section{Genetic and Clinical Factors}

Interpatient variability in warfarin dose can also be partially be explained by patient-specific factors, such as age, body surface area, and illness. ${ }^{26}$ Patients who consume large amount of dietary vitamin $\mathrm{K}$ also require significantly higher warfarin doses to prevent subtherapeutic dosing. ${ }^{27}$ Additionally, medications that impact warfarin pharmacokinetics or pharmacodynamics can also dramatically alter dose requirement. ${ }^{28}$ For example, drug inhibition of the warfarin metabolizing enzyme CYP2C9 can alter warfarin pharmacokinetics, resulting in a decreased warfarin dose requirement. ${ }^{28}$ In patients receiving multiple concomitant medications, the association between genetic variants and warfarin dose is reduced because of the additional nongenetic variability in dose requirements introduced by the effect of these drugs on warfarin metabolism. ${ }^{29,30}$ The relative importance of genetics for predicting warfarin dose can therefore be modulated by environmental factors, which should be taken into consideration when estimating warfarin dose requirements.

\section{Scope and Purpose}

The purpose of this review is to provide clinical practice recommendations on both the prospective utility of pharmacogenetic testing and on genotype-specific treatment options to enable medical professionals to make more informed decisions about optimal warfarin therapy. In particular, this document addresses the following key questions:

1. In treatment of patients with an indication for warfarin, does the use of genetic testing before initiation of warfarin, when compared with no testing, improve achievement of stable anticoagulation and reduce adverse effects?

2. Are there subpopulations of patients where testing will be less beneficial/more beneficial compared with others?

3. How should patients with an indication for warfarin therapy be managed based on their genotyping results?

By addressing these key questions, we hope to clearly define how genetic testing should be incorporated into warfarin therapy to gain maximum benefits and limit patient harm. The recommendations provided should be interpreted in the context of the unique clinical circumstances for each patient.

429 


\section{METHODS}

A standard guideline development process was followed in accordance with the quality criteria suggested by the Appraisal of Guidelines Research and Evaluation Enterprise (AGREE), an international endeavor aimed at improving the quality of practice guidelines. ${ }^{31,32}$ This process involved a systematic literature search followed by critical appraisal of the retrieved evidence (Table 2). Clinical practice recommendations were developed during a workshop meeting of guideline development group members. Recommendations were assigned 1 of 3 levels based on the strength of scientific evidence on which the recommendations were formed, as well as the balance between benefits and risks for genotype-guided therapy (Table 3). Draft guideline documents were submitted to a tiered review process, which included internal review by the guideline development group members, followed by external review both by content experts and by members of the intended target audience. Additional details on the literature search strategy, evidence review and appraisal, and recommendation development are provided in the Supplemental Digital Content 1 (see Data, http://links.lww.com/ TDM/A107).

\section{CLINICAL PRACTICE RECOMMENDATIONS}

\section{Should Pharmacogenetic Testing Be Performed in Warfarin-Naive Patients Before Initiation of Warfarin Therapy?}

Testing of all warfarin-naive patients for VKORC1 $(-1639 \mathrm{G}>\mathrm{A}), C Y P 2 C 9^{*} 2$, and $C Y P 2 C 9^{*} 3$ should be considered before initiation of therapy and within the first 2 weeks of therapy (level B-Table 3). Genetic testing for $C Y P 2 C 9^{*} 5$, $* 6$, *8, or *11 and CYP4F2 V433M is currently not recommended (C).

TABLE 2. Grading Scheme Used for Critical Appraisal of Evidence

\begin{tabular}{ccc}
\hline Grade & Results & Description \\
\hline++++ & Consistent, generalizable & $\begin{array}{c}\text { Strong general conclusions can } \\
\text { be drawn that are } \text { unlikely to } \\
\text { change based on further } \\
\text { research }\end{array}$ \\
& & Evidence allows general \\
+++ & Consistent, but limited & conclusions, but with \\
& quantity, quality, or & reduced confidence; further \\
& generalizability & research is likely to have an \\
& & important impact on \\
& & confidence in conclusions \\
& Inconsistent or insufficient & No general conclusions can be \\
& quantity/quality, & drawn or conclusions are \\
& encouraging & likely to change based on \\
& & further research, but current \\
& & evidence is encouraging \\
& Inconsistent or insufficient & No conclusions can be drawn \\
& quantity/quality, & or conclusions are likely to \\
& discouraging & change based on future \\
& studies, and current evidence \\
& is discouraging
\end{tabular}

TABLE 3. Grading Scheme Used for Clinical Practice Recommendations

\begin{tabular}{|c|c|c|}
\hline Level & Strength & Evidence Basis \\
\hline A & Strong & $\begin{array}{l}\text { Based on strong scientific evidence, } \\
\text { benefits clearly outweigh risks }\end{array}$ \\
\hline B & Moderate & $\begin{array}{l}\text { Based on reduced confidence } \\
\text { scientific evidence and expert } \\
\text { opinion, benefits likely to outweigh } \\
\text { risks }\end{array}$ \\
\hline $\mathrm{C}$ & Optional & $\begin{array}{l}\text { Based mainly on expert opinion, for } \\
\text { use with evidence development in } \\
\text { a research context }\end{array}$ \\
\hline
\end{tabular}

\section{Considerations}

Carriers of $V K O R C 1(-1639 \mathrm{G}>\mathrm{A}), C Y P 2 C 9^{*} 2$, and $C Y P 2 C 9 * 3$ require a significantly lower warfarin dose compared with wild-type carriers. They also require less time to reach a therapeutic INR, are more likely to become overanticoagulated (INR $\geq 4$ ), and are at greater risk of experiencing an adverse event. There is inconsistent but encouraging evidence that pharmacogenetic-guided dosing increases time within the therapeutic range and reduces hospitalization rates of patients on warfarin therapy.

Testing should be performed before initiating therapy. If this is not feasible, we recommend that testing be considered if test results can be obtained within the first 2 weeks of therapy, as genetic information can still be useful for estimating the maintenance dose. After 2 weeks, the benefits derived from genetic testing are reduced.

At this time, we do not recommend testing for CYP $2 C C^{*} 5, * 6, * 8$, or $* 11$ for any patients. Although there is strong evidence of an association between these variants and required dose in African Americans, it remains unclear how influential these single-nucleotide polymorphisms are when other CYP2C9 and VKORC1 genotypes are considered and how best to determine an appropriate dose when this information is available as they were not included in most studies evaluating genotype-guided dosing algorithms. However, if $C Y P 2 C 9 * 5$ or $* 6$ genotype is already known for a specific patient, they can be incorporated into the dosing algorithm at www.WarfarinDosing.org. We make the same recommendation for testing of CYP4F2 V433M, which can also be entered into the web-based dosing algorithm.

\section{Are There Subpopulations of Patients Where Testing Will Be Less Beneficial/More Beneficial Compared With Others?}

Testing should be considered for all patients who are at increased risk of bleeding complications, who consistently show out-of-range INRs, or suffer adverse events while receiving warfarin (B). We also recommended that testing be considered for all pediatric patients (B). Testing is not recommended in patients who have reached a stable INR (A).

\section{Considerations}

For the majority of patients, we do not recommend genetic testing once a stable INR has been reached. However, genetic testing should be performed if patients consistently 
show out-of-range INR values or fluctuations in INR values for more than 3-4 weeks that are not accounted for by other variables (eg, elderly age, elevated baseline INR, hypoalbuminemic patients, impaired nutrition, heart failure, concomitant medications that increase sensitivity to warfarin, dietary measures). Genetic testing should also be considered for patients who are at increased risk of bleeding complications. Specific risk factors for bleeding include older age, history of gastrointestinal (GI) tract bleeding, hypertension, cerebrovascular disease, serious heart disease, anemia, malignancy, trauma, renal insufficiency, recent percutaneous coronary intervention, new onset atrial fibrillation, and patients who are receiving triple therapy (clopidogrel, aspirin, and warfarin). ${ }^{33-35} \mathrm{We}$ also recommend testing patients who suffer bleeding events while on warfarin therapy, as this may help to determine whether the underlying cause of bleeding was likely due to diet, concomitant medications or other clinical factors, or as a result of genetic variants. In this instance, discerning the reason for the bleeding episode could help prevent future bleeding events and allow for safer management of these patients overall.

Emerging evidence suggests a strong association between genetic variants and required warfarin dose and warfarin-related outcomes in children. Although the impact of genotype-guided dosing on clinical outcomes has not yet been studied in children, we recommend that testing also be considered for all pediatric patients within the first 2 weeks of warfarin therapy because of consistent genetic associations (level B).

\section{How Should Patients With an Indication for Warfarin Therapy Be Managed Based on Their Genotyping Results?}

Genotyping results should be interpreted using a pharmacogenetic dosing algorithm to estimate the required dose (A). Several pharmacogenetic dosing algorithms are available for use, including algorithms accessible online at www. warfarindosing.org and as an iWarfarin application.

After testing for $C Y P 2 C 9^{*} 2, C Y P 2 C 9^{*} 3$, and $V K O R C 1$ $(-1639 \mathrm{G}>\mathrm{A})$, pharmacogenetic dosing algorithms that incorporate both clinical variables and genetic information should be used to predict a stable warfarin dose. These include the International Warfarin Pharmacogenetics Consortium (IWPC) model, ${ }^{36}$ which is available as the iPhone/iPad/ iPod application iWarfarin, ${ }^{37}$ and the updated Gage et al model found at www.WarfarinDosing.org. These algorithms have previously been shown to posses the greatest predictive ability and are easily accessible for clinicians. ${ }^{38-40}$

We recommend using the FDA-approved dosing table provided on the warfarin label insert (see Table 3, Supplemental Digital Content 1, http://links.lww.com/TDM/A107) when physicians are unable to access pharmacogenetic dosing algorithms. These dose recommendations are derived from a large database containing both clinical and genetic information; however, the table does not incorporate relevant patientspecific clinical factors. Given that clinical factors also significantly affect warfarin dose, dosing algorithms that consider both genetic and clinical information should be preferred. ${ }^{41}$
There are several pediatric-derived dosing algorithms that have also been published. ${ }^{42-46} \mathrm{We}$ recommend using the model developed by Biss et al, as the accuracy of this model has been validated using retrospective data in replication cohorts. ${ }^{44,47,48}$ To date, none of the pediatric dosing algorithms have been evaluated in prospective cohorts and are not available as a ready-to-use computer/phone application or Web site. Alternatively, the IWPC model has also been shown to be predictive of maintenance dose in children, although it is less accurate compared with pediatric-derived pharmacogenetic dosing models. ${ }^{43,44}$

Currently, validated genetic tests are not routinely available in some hospitals or outpatient laboratories. For those that offer these tests, the turnaround time for test results that meet the requirements set forth by Clinical Laboratory Standards Institute is approximately 5-10 days (eg, Mayo and Arup laboratories). The technology is available where the analytical turnaround time is about 24 hours, thus improvement in turnaround time is expected. For now, healthcare providers should consult their local laboratories for test availability and turnaround time to optimize patient management. A list of testing laboratories is provided in the Supplemental Digital Content 1 (see Table 4, http://links.lww.com/TDM/A107). We do not recommend delaying therapy for test results in cases where anticoagulation is required immediately. Rather, depending on when test results are available, they can be incorporated into a dose-refinement algorithm after the initiation of therapy (eg, WRAPID, www.warfarindosing.org).

Of importance, pharmacogenetic-guided dosing should not replace regular INR monitoring. Rather, test results should be used to help physicians estimate an appropriate warfarin dose, while still using regular INR monitoring to ensure that stable anticoagulation is achieved. In all situations, informed decision making and informed consent are required by patients after counseling on the use, benefits and risks, and implications of these tests by the healthcare provider.

\section{EVIDENCE SUMMARY}

A detailed description of supporting evidence is provided in the Supplemental Digital Content 1 (see Data, http://links.lww.com/TDM/A107). Here, we provide a brief summary and appraisal of the evidence for certain outcomes investigated.

\section{Warfarin Dose Is Correlated With CYP2C9 and VKORC1 Genotypes}

There is strong evidence $(++++$ evidence, Table 2$)$ that the required warfarin dose is associated with VKORC1-1639, $C Y P 2 C 9^{*}$, and $C Y P 2 C 9^{*} 3$ variants (see Table 6, Supplemental Digital Content 1, http://links.lww.com/TDM/A107). This is based on a large number of studies showing consistent results. Importantly, these studies were comprised a diverse patient population, including African Americans, Caucasians, Japanese, Han Chinese, Hispanics, Indians, and several other ethnicities. The majority of studies (39 of 47) found that VKORC1 is the most important genetic factor influencing variability in warfarin dosing (Table 4). When comparing ethnic populations, this variant accounts for the largest amount of 
TABLE 4. Variability in Warfarin Dose Explained by VKORC1 and CYP2C9 Genotypes in Different Ethnic Populations

\begin{tabular}{lccccc}
\hline Genotype & Caucasian, \% & East Asian, \% & West Asian, \% & Hispanic, \% & African/African American, \% \\
\hline$V K O R C 1-1639 \mathrm{G}>\mathrm{A}$ & $13.8-47^{49,65}$ & $19.8-35.5^{66,67}$ & $20.3-34.1^{68,69}$ & $11.3-30.3^{70,71}$ & $4-9^{15,72}$ \\
CYP2C $9 * 2$ or $* 3$ & $2-27^{29,42}$ & $3.4-11.2^{73,74}$ & $8.1-19.0^{69,75}$ & $7.1-8^{70,71}$ & $<5.6^{15,16}$ \\
CYP $2 C 9 * 5, * 6, * 8, * 11$ & NA & NA & NA & NA & $<5-6^{16,76}$ \\
\hline
\end{tabular}

NA, not available.

dose variability in East and West Asians and the least amount in African Americans (Table 4). Up to $47 \%$ of the variation in warfarin dosing can be explained by the VKORC1 genotype (Table 4). ${ }^{49}$

CYP2C9 is the second strongest genetic factor associated with warfarin dose, ${ }^{50}$ accounting for approximately $2 \%-27 \%$ of the variability (Table 4). ${ }^{29,42}$ The $* 2$ and $* 3$ variants are most frequent in Caucasians and contribute to the larger impact on dose variability in this population compared with other populations (Table 4). ${ }^{20}$ Accordingly, the amount of variability explained by $C Y P 2 C 9 * 2$ and $* 3$ is less in African American and Asians, likely due to the lower frequency of these alleles.

\section{Association Between Warfarin Dose and CYP4F2 Genotype}

The overall evidence grading for an association between CYP4F2 genotype and required warfarin dose is moderate (++evidence). This grading is based on inconsistent results across studies, particularly regarding the amount of variability explained by CYP4F2 (ranges from $1 \%$ to $11 \%$ ). ${ }^{51,52}$ Moreover, the clinical utility of including $C Y P 4 F 2$ in dosing models that already include VKORC1 and CYP2C9 remains unclear. Further studies are required to determine the clinical benefit of incorporating this variant into a dosing algorithm.

\section{Stable Warfarin Dose Is More Accurately Predicted by Genotype-Guided Dosing Compared With Standard Dosing or Clinical Algorithms}

There is strong evidence (++++, Table 2) that pharmacogenetic dosing algorithms more accurately predict the stable warfarin dose when compared with standard empirical dosing or clinical dosing algorithms. This is based on consistent findings across all studies included in the evidence summary.

The pharmacogenetic dosing algorithms most commonly tested were those developed by Gage et al, ${ }^{53}$ Sconce et $a 1,{ }^{54}$ and the IWPC. ${ }^{36}$ When Sagreiya et ${ }^{38}{ }^{38}$ compared these 3 algorithms, as well as an additional 4 algorithms, with the IWPC clinical algorithm, they found that the models of IWPC pharmacogenetics and Gage et al possessed the greatest predictive ability and performed very similarly (IWPC: $\mathrm{R}^{2}=0.50$, Gage: $\mathrm{R}^{2}=0.49$, Clinical: $\mathrm{R}^{2}=22$ ). Furthermore, all pharmacogenetic models outperformed the IWPC clinical model in terms of $\mathrm{R}^{2}$ and mean absolute error values when predicting the required dose.

A novel pharmacogenetics-guided initiation and maintenance dosing regimen was also investigated in a prospective cohort study (WRAPID; see Table 5, Supplemental Digital Content 1, http://links.lww.com/TDM/A107). ${ }^{39}$ As INRs became available, they were entered into the algorithm, along with clinical variables and genetics, to determine an appropriate maintenance dose. When the algorithm was applied to all study participants, the proportion of variance explained by the maintenance dose regression model was $42 \%$, whereas the variance explained after INR-guided dose adjustments was $70 \%$. Therefore, prediction of maintenance dose may be further improved by incorporating initiation INRs into a geneticsbased dosing algorithm.

\section{Time to Therapeutic/Stable INR}

Time to therapeutic or stable INR is frequently used as a clinical end point because once a stable INR is reached, patients are less likely to show fluctuations in subsequent INR measurements and the risk of bleeding is significantly reduced. ${ }^{55}$ When evaluating this outcome, both observational and intervention studies were considered. The overall evidence grading for association between CYP2C9 and VKORC1 genotypes and time to first therapeutic INR is fairly strong (+++ evidence). This is based on consistent results from a large number of studies. However, the association between genetic variants and time required to reach stable INR, defined as consecutive INRs in the therapeutic range, is less consistent and needs to be evaluated further. Based on results from intervention studies, the evidence for genotype-guided dosing to reduce the amount of time required to reach therapeutic/stable INR is inconsistent (++evidence). Six of the 11 studies that prospectively compared standard dosing practices with genotype-guided dosing found that the time to reach therapeutic INR was significantly shorter in the genotype-guided group. All studies evaluated were randomized controlled trials (RCTs), with the exception of 1 study in which every patient was dosed using a pharmacogenetics-based dosing model. Because of a possibility of "over management" of patients, the results may not reflect real-world outcomes. Based on inconsistent findings and differences in study quality and the comparison groups evaluated, no general conclusions can be drawn.

\section{Time Within the Therapeutic INR Range}

The strength of evidence for an association between genotype and time spent in the therapeutic INR range for the duration of warfarin therapy is low (+evidence). This is mainly due to a limited quantity of studies $(n=3)$ and a lack of significant findings. However, the evidence for genotypeguided dosing to increase the amount of time spent in the therapeutic range is insufficient but encouraging (++evidence). All intervention studies reported that patients who received pharmacogenetic-guided dosing spent more time in the therapeutic range compared with the control group; 
however, there were inconsistencies in the magnitude of effect and not all findings were statistically significant. Based on studies that reported a significant finding, genotype-guided dosing increased the amount of time within the therapeutic range by $7 \%-21 \%$ compared with using empirical dosing or clinical dosing algorithms. Nevertheless, no general conclusions can be drawn at this time.

\section{Time to INR $\geq 4$ or Incidence/Frequency of INR $\geq 4$}

The incidence/frequency of INR measurements $\geq 4$ is often used as a surrogate marker for the risk of bleeding complications during warfarin therapy. ${ }^{56}$ The evidence grading for an association between $C Y P 2 C 9$ and $V K O R C 1$ genotype and incidence of INR $\geq 4$ is fairly strong ( +++ evidence). This is due to a large number of studies reporting consistent results. However, based on the available evidence, it is unclear whether genotype is associated with incidence of INR $\geq 4$ in African Americans and whether being heterozygous for $V K O R C 1$ also increases the risk of INR $\geq 4$. Only 1 observational study investigated time to INR $\geq 4$ and found that carriers of $C Y P 2 C 9 / V K O R C 1$ variants required significantly less time to reach an INR $>4$ compared with noncarriers, representing a risk for overanticoagulation. ${ }^{57}$ More evidence is needed before this association can be comprehensively assessed. In contrast, the evidence grading for genotype-guided dosing to decrease the incidence or time to INR $\geq 4$ is low (+evidence). Four of 5 studies reported no difference in incidence/time to INR $\geq 4$ between genotypeguided dosing and control patients. One study that used pharmacogenetic dosing in all patients did not find a significant difference in time to INR $>4$ between genotype groups, suggesting an elimination of genotype-related differences that have been reported in observational studies. ${ }^{39}$

\section{Incidence of Adverse Events}

There is fairly strong evidence that CYP2C9 and VKORC1 genotypes are associated with warfarin-induced adverse events (+++evidence). Specifically, 14 of 17 studies reported a significant association between genotypes and incidence of bleeding events. The association is strongest with CYP2C9 variant genotypes, whereas there is less evidence of an association with the VKORC1 genotype. Further research is required to determine the role of $V K O R C 1$ in warfarininduced bleeding events. In prospective studies that investigated warfarin-induced adverse events, there is insufficient evidence (++evidence) that genotype-guided dosing can decrease the number of adverse events. With the exception of 1 study, it was consistently shown that there is a trend toward less adverse events in the genotype-guided dosing group, suggesting that the rarity of warfarin-induced adverse events may contribute to nonsignificant findings rather than a lack of association. However, 1 study used historical controls, whereas another study combined adverse events with other clinical outcomes for analyses, potentially biasing the results in favor of genotype-guided dosing. Overall, the lack of consistent significant findings weakens the evidence for this outcome.

\section{FUTURE DIRECTIONS}

Pharmacogenetic dosing algorithms have been shown to predict warfarin doses to a certain degree of confidence. However, their ability to improve clinical outcomes (eg, decrease the number of bleeding events) remains to be confirmed with a greater level of confidence. In 2013, 2 large RCTs investigating the clinical benefit of genotypebased warfarin dosing were published but provided different conclusions. The EU-PACT $\operatorname{trial}^{58}(\mathrm{n}=455)$ reported that genotype-based dosing was associated with a higher percentage of time in the therapeutic range compared with standard dosing $(67.4 \%$ versus $60.3 \%, P<0.001)$, whereas the COAG $\operatorname{trial}^{59}(\mathrm{n}=1015)$ found that there was no difference between genotype-guided dosing and dose determined using a clinical algorithm when investigating the same primary outcome $(45.2 \%$ versus $45.4 \% ; P=0.91)$. Some key differences between the 2 studies were length of follow-up time (12 weeks for EU-PACT and 4 weeks for COAG), determination of dose in the nongenotype group (fixed dose in EUPACT and clinical dosing algorithm in COAG), patient ancestry (2\% non-Caucasian in EU-PACT and 33\% nonCaucasian in COAG), and the availability of genetic test results (EU-PACT genotype results were available in approximately 2 hours, COAG genotype results were not available before the first dose for $55 \%$ of patients). These differences in study design may have largely impacted the primary outcomes.

These studies also raise questions about the ability of RCTs to reflect real-world clinical settings and whether studies conducted in very controlled settings and specialized centers are appropriate for determining the clinical utility of pharmacogenetic testing in warfarin therapy. It has previously been noted that in the case of warfarin RCTs, patients in the control group may be "over managed" compared with standard clinical care, diluting the anticipated effect of genotype-based dosing on clinical outcomes. An alternative approach could be a prospective nonrandomized study where genetic testing is incorporated into clinical care and compared with standard dosing practices in a more realistic setting to better determine the utility of genetic testing. ${ }^{60-62}$ Two ongoing RCTs [WARFARIN Study (NCT01305148) and GIFT (NCT01006733)] are using incidence of adverse events, including bleeding and thromboembolism, as the primary study outcomes. This is in contrast to COAG and EU-PACT, which used time in therapeutic range as a primary outcome. Using a more clinically relevant outcome such as adverse events may also help to more accurately represent the true clinical utility of genotype-guided dosing, as well as give a better understanding of the relative importance of $V K O R C 1$ and $C Y P 2 C 9$ variants in the context of warfarin ADRs. The design of future prospective interventional studies should also consider newly discovered factors influencing warfarin dose, including novel genetic variants in African Americans and differences in response between patient groups [eg, venous thromboembolism (VTE) versus atrial fibrillation], ${ }^{63}$ as well as differences between clinical settings to better evaluate the clinical utility of genotype-guided warfarin dosing. 
Table 5 summarizes research gaps that require further consideration. Specifically, future research should aim to improve the accuracy of pharmacogenetic dosing algorithms in non-European patients. This could include incorporating additional CYP2C9 and VKORC1 variants into dosing models to account for interethnic differences in genotype frequency and dose requirements or genome-wide association studies in homogeneous patient cohorts to discover novel genetic markers that are ethnic specific. Optimization of dosing algorithms in patients with a target INR range outside of $2-3$ is also needed.

Genotype-based initiation dose algorithms, such as those developed by Gong et $\mathrm{al}^{39}$ and Avery et $\mathrm{al}^{40}{ }^{40}$ also require further investigation. The use of initiation doses may be especially important in VTE patients, as these patients have shown to be more resistant to warfarin's therapeutic effect when compared with atrial fibrillation patients..$^{13}$ However, there is concern over using initiation doses, as some patients may become overanticoagulated and be at higher risk of bleeding. More information is required regarding the clinical utility of genetics-based initiation dose algorithms and whether these algorithms are more beneficial for specific patient populations that have historically required higher warfarin doses (eg, VTE patients).

Currently, approximately $50 \%$ of the dose variation can be explained by clinical and genetic factors combined. Potential sources of missing information include additional genetic variation, epigenetic factors, additional drug-drug interactions, and patient behaviors, including diet, exercise, and compliance. ${ }^{64}$ Future pharmacogenetic studies that are able to control for environmental factors may reveal additional genetic variants implicated in warfarin dosing.

The investigation of rare variants in CYP2C9 and VKORC1 may also improve the prediction of extreme phenotypes (extremely high warfarin sensitivity or warfarin resistance). Rost et $\mathrm{al}^{21}$ identified 4 VKOR variants that confer reduced catalytic activity and are associated with "warfarin resistance." Further studies are required to elucidate the mechanisms behind extreme phenotypes and the predictive value of these rare variants.

New oral anticoagulants (NOACs) are now available as a possible alternative to warfarin and offer some advantages,

TABLE 5. Knowledge Gaps That Warrant Future Research

Improving dosing algorithms in non-European patients

- Incorporation of additional CYP2C9 variants

Additional prospective interventional studies to assess clinical utility of genotype-guided warfarin dosing for different indications, clinical settings, ethnicities

Optimization of dosing algorithms for patients with target INR outside of 2-3

Further validation of genotype-based initiation doses and algorithms

Identify additional sources of variability in dose requirement

- Additional genetic factors

- Epigenetic factors

- Patient behavior (eg, diet, exercise, compliance)

Elucidate mechanisms underlying extreme phenotypes, eg, rare SNPs in CYP2C9 and VKORC1

Benefit of genotype-guided warfarin therapy in the context of NOACs including rapid onset and lack of need for monitoring. However, the paucity of information regarding safety and effectiveness of NOACs warrants further research and longterm evaluation of these compounds. It is expected that as new compounds emerge, warfarin will remain an important and frequently used drug given its proven efficacy, low cost, and years of physician experience compared with NOACs.

\section{ACKNOWLEDGMENTS}

We would like to thank all members of the Canadian Pharmacogenomics Network for Drug Safety (CPNDS) clinical recommendation group, who participated in the clinical practice recommendation workshop or reviewed the draft document: Vancouver, BC, Canada: University of British Columbia, BC Children's Hospital, Child and Family Research Institute: Bruce Carleton, Michael R. Hayden, Ursula Amstutz, Soomi Hwang, Martin Hosking, Sean Virani, Colin J. Ross, Stuart MacLeod, Rod Rassekh, Anne Smith, Liam Brunham; ON, Canada: Sunnybrook Health Sciences Centre: Neil H. Shear; Hospital for Sick Children: Gideon Koren, Shinya Ito; Ontario Cancer Institute: Geoffrey Liu. London, ON, Canada: University of Western Ontario and London Health Sciences Centre: Michael J. Rieder, Richard Kim. Ottawa, ON, Canada: Department of National Defense: Maurica Maher. Montréal, QC, Canada: Université de Montréal: Jacques Turgeon, Véronique Michaud. Indianapolis, IN: Indiana University: David Flockhart. Orlando, FL: University of Florida: Lawrence Lesko, Jenna Rhoades. We would also like to thank Tricia Yu from the UBC Eric Hamber Library for reviewing the literature search strategy and Gabriella Groeneweg for her contributions to the management of this project and the organization of the recommendation development workshop.

\section{REFERENCES}

1. Ansell J, Hirsh J, Hylek E, et al. Pharmacology and management of the vitamin $\mathrm{K}$ antagonists: american college of chest physicians evidencebased clinical practice guidelines (8th edition). Chest. 2008;133: 160S-198S.

2. McWilliam A, Lutter R, Nardinelli C. Health Care Savings from Personalizing Medicine Using Genetic Testing: The Case of Warfarin. Washington, DC: American Enterprise Institute-Brookings Joint Center for Regulatory Studies; 2006.

3. Kaminsky LS, Zhang ZY. Human P450 metabolism of warfarin. Pharmacol Ther. 1997;73:67-74.

4. Wallin R, Hutson SM. Warfarin and the vitamin K-dependent gammacarboxylation system. Trends Mol Med. 2004;10:299-302.

5. King CR, Deych E, Milligan P, et al. Gamma-glutamyl carboxylase and its influence on warfarin dose. Thromb Haemost. 2010;104:750-754.

6. Fang MC, Chang YC, Hylek EM, et al. Advanced age, anticoagulation intensity, and risk for intracranial hemorrhage among patients taking warfarin for atrial fibrillation. Ann Intern Med. 2004;141:745-752.

7. Hylek EM, Singer DE. Risk factors for intracranial hemorrhage in outpatients taking warfarin. Ann Intern Med. 1994;120:897-902.

8. Linkins LA, Choi PT, Douketis JD. Clinical impact of bleeding in patients taking oral anticoagulant therapy for venous thromboembolisma meta-analysis. Ann Intern Med. 2003;139:893-900.

9. Da Silva M, Sobel M. Anticoagulants: to bleed or not to bleed, that is the question. Semin Vasc Surg. 2002;15:256-267.

10. Budnitz DS, Pollock DA, Weidenbach KN, et al. National surveillance of emergency department visits for outpatient adverse drug events. $J \mathrm{Am}$ Med Assoc. 2006;296:1858-1866. 
11. Wysowski DK, Nourjah P, Swartz L. Bleeding complications with warfarin use - a prevalent adverse effect resulting in regulatory action. Arch Intern Med. 2007;167:1414-1419.

12. Sistonen J, Fuselli S, Palo JU, et al. Pharmacogenetic variation at CYP2C9, CYP2C19, and CYP2D6 at global and microgeographic scales. Pharmacogenet Genom. 2009;19:170-179.

13. Gong IY, Schwarz UI, Crown N, et al. Clinical and genetic determinants of warfarin pharmacokinetics and pharmacodynamics during treatment initiation. PLoS One. 2011;6:e27808. doi: 10.1371/journal. pone. 0027808 .

14. Lee CR, Goldstein JA, Pieper JA. Cytochrome P450 2C9 polymorphisms: a comprehensive review of the in-vitro and human data. Pharmacogenetics. 2002;12:251-263.

15. Perera MA, Gamazon E, Cavallari LH, et al. The missing association: sequencing-based discovery of novel SNPs in VKORC1 and CYP2C9 that affect warfarin dose in African Americans. Clin Pharmacol Ther. 2011;89:408-415.

16. Cavallari LH, Langaee TY, Momary KM, et al. Genetic and clinical predictors of warfarin dose requirements in African Americans. Clin Pharmacol Ther. 2010;87:459-464.

17. Limdi NA, Wiener H, Goldstein JA, et al. Influence of CYP2C9 and VKORC1 on warfarin response during initiation of therapy. Blood Cells Mol Dis. 2009;43:119-128.

18. Scott SA, Jaremko M, Lubitz SA, et al. CYP2C9*8 is prevalent among African-Americans: implications for pharmacogenetic dosing. Pharmacogenomics. 2009;10:1243-1255.

19. Rieder MJ, Reiner AP, Gage BF, et al. Effect of VKORC1 haplotypes on transcriptional regulation and warfarin dose. $N$ Engl J Med. 2005;352: 2285-2293.

20. Kersey PJ, Allen JE, Christensen M, et al. Ensembl genomes 2013: scaling up access to genome-wide data. Nucleic Acids Res. 2014;42: D546-D552.

21. Rost S, Fregin A, Ivaskevicius V, et al. Mutations in VKORC1 cause warfarin resistance and multiple coagulation factor deficiency type 2 . Nature. 2004;427:537-541

22. Harrington DJ, Underwood S, Morse C, et al. Pharmacodynamic resistance to warfarin associated with a Val66Met substitution in vitamin $\mathrm{K}$ epoxide reductase complex subunit 1. Thromb Haemost. 2005;93:23-26.

23. Loebstein R, Dvoskin I, Halkin H, et al. A coding VKORC1 Asp36Tyr polymorphism predisposes to warfarin resistance. Blood. 2007;109: $2477-2480$.

24. Takahashi H, Wilkinson GR, Nutescu EA, et al. Different contributions of polymorphisms in VKORC1 and CYP2C9 to intra- and interpopulation differences in maintenance dose of warfarin in Japanese, Caucasians and African-Americans. Pharmacogenet Genom. 2006;16: 101-110.

25. Caldwell MD, Awad T, Johnson JA, et al. CYP4F2 genetic variant alters required warfarin dose. Blood. 2008;111:4106-4112.

26. Kim MJ, Huang SM, Meyer UA, et al. A regulatory science perspective on warfarin therapy: a pharmacogenetic opportunity. J Clin Pharmacol. 2009;49:138-146.

27. Lubetsky A, Dekel-Stern E, Chetrit A, et al. Vitamin K intake and sensitivity to warfarin in patients consuming regular diets. Thromb Haemost. 1999;81:396-399.

28. Heimark LD, Wienkers L, Kunze K, et al. The mechanism of the interaction between amiodarone and warfarin in humans. Clin Pharmacol Ther. 1992;51:398-407.

29. Herman D, Locatelli I, Grabnar I, et al. Influence of CYP2C9 polymorphisms, demographic factors and concomitant drug therapy on warfarin metabolism and maintenance dose. Pharmacogenomics J. 2005;5: 193-202.

30. Michaud V, Vanier MC, Brouillette D, et al. Combination of phenotype assessments and CYP2C9-VKORC1 polymorphisms in the determination of warfarin dose requirements in heavily medicated patients. Clin Pharmacol Ther. 2008;83:740-748.

31. Brouwers MC, Kho ME, Browman GP, et al. AGREE II: advancing guideline development, reporting and evaluation in health care. CMAJ. 2010;182:E839-E842.

32. Amstutz U, Shear NH, Rieder MJ, et al. Recommendations for HLAB*15:02 and HLA-A*31:01 genetic testing to reduce the risk of carbamazepine-induced hypersensitivity reactions. Epilepsia. 2014;55: 496-506.
33. Ridker PM, Goldhaber SZ, Danielson E, et al. Long-term, low-intensity warfarin therapy for the prevention of recurrent venous thromboembolism. $N$ Engl J Med. 2003;348:1425-1434.

34. Beyth RJ, Quinn LM, Landefeld CS. Prospective evaluation of an index for predicting the risk of major bleeding in outpatients treated with warfarin. Am J Med. 1998;105:91-99.

35. Wells PS, Forgie MA, Simms M, et al. The outpatient bleeding risk index: validation of a tool for predicting bleeding rates in patients treated for deep venous thrombosis and pulmonary embolism. Arch Intern Med. 2003;163:917-920.

36. Klein TE, Altman RB, Eriksson N, et al. Estimation of the warfarin dose with clinical and pharmacogenetic data. $N$ Engl J Med. 2009;360: 753-764.

37. The Institute for Pharmacogenomics and Individualized Therapy. iWarfarin. (IPhone application). Version 1.0. Available at Apple Application Store: http://ax.itunes.apple.com/us/app/iwarfarin/id359516751?mt=8

38. Sagreiya $\mathrm{H}$, Berube $\mathrm{C}$, Wen $\mathrm{A}$, et al. Extending and evaluating a warfarin dosing algorithm that includes CYP4F2 and pooled rare variants of CYP2C9. Pharmacogenet Genom. 2010;20:407-413.

39. Gong IY, Tirona RG, Schwarz UI, et al. Prospective evaluation of a pharmacogenetics-guided warfarin loading and maintenance dose regimen for initiation of therapy. Blood. 2011;118:3163-3171.

40. Avery PJ, Jorgensen A, Hamberg AK, et al. A proposal for an individualized pharmacogenetics-based warfarin initiation dose regimen for patients commencing anticoagulation therapy. Clin Pharm Ther. 2011;90: 701-706.

41. Finkelman BS, Gage BF, Johnson JA, et al. Genetic warfarin dosing tables versus algorithms. J Am Coll Cardiol. 2011;57:612-618.

42. Moreau C, Bajolle F, Siguret V, et al. Vitamin K antagonists in children with heart disease: height and VKORC1 genotype are the main determinants of the warfarin dose requirement. Blood. 2012;119:861-867.

43. Biss TT, Avery PJ, Brandao LR, et al. VKORC1 and CYP2C9 genotype and patient characteristics explain a large proportion of the variability in warfarin dose requirement among children. Blood. 2012;119:868-873.

44. Shaw K, Amstutz U, Hildebrand C, et al. VKORC1 and CYP2C9 genotypes are predictors of warfarin-related outcomes in children. Pediatr Blood Cancer. 2014;61:1055-1062.

45. Vear SI, Ayers GD, Van Driest SL, et al. The impact of age and CYP2C9 and VKORC1 variants on stable warfarin dose in the paediatric population. Br J Haematol. 2014;165:832-835.

46. Hamberg AK, Wadelius M, Friberg LE, et al. Characterising variability in warfarin dose requirements in children using modelling and simulation. Br J Clin Pharmacol. 2014;78:158-169.

47. Hamberg AK, Friberg LE, Hanseus K, et al. Warfarin dose prediction in children using pharmacometric bridging-comparison with published pharmacogenetic dosing algorithms. Eur J Clin Pharmacol. 2013;69: $1275-1283$.

48. Biss T, Hamberg AK, Avery $\mathrm{P}$, et al. Warfarin dose prediction in children using pharmacogenetics information. Br J Haematol. 2012;159:106-109.

49. D'andrea G, D'Ambrosio RL, Di Perna P, et al. A polymorphism in the VKORC1 gene is associated with an interindividual variability in the dose-anticoagulant effect of warfarin. Blood. 2005;105:645-649.

50. Takeuchi F, McGinnis R, Bourgeois S, et al. A genome-wide association study confirms VKORC1, CYP2C9, and CYP4F2 as principal genetic determinants of warfarin dose. PLoS Genet. 2009;5:e1000433. doi: 10. 1371/journal.pgen.1000433

51. Singh O, Sandanaraj E, Subramanian K, et al. Influence of CYP4F2 rs2108622 (V433M) on warfarin dose requirement in Asian patients. Drug Metab Pharmacokinet. 2011;26:130-136.

52. Perini JA, Struchiner CJ, Silva-Assuncao E, et al. Impact of CYP4F2 rs2108622 on the stable warfarin dose in an admixed patient cohort. Clin Pharmacol Ther. 2010;87:417-420.

53. Gage BF, Eby C, Johnson JA, et al. Use of pharmacogenetic and clinical factors to predict the therapeutic dose of warfarin. Clin Pharmacol Ther. 2008;84:326-331

54. Sconce EA, Khan TI, Wynne HA, et al. The impact of CYP2C9 and VKORC1 genetic polymorphism and patient characteristics upon warfarin dose requirements: proposal for a new dosing regimen. Blood. 2005; 106:2329-2333.

55. Hirsh J, Fuster V, Ansell J, et al. American Heart Association/American College of Cardiology Foundation guide to warfarin therapy. Circulation. 2003;107:1692-1711 
56. Ansell J, Hirsh J, Dalen J, et al. Managing oral anticoagulant therapy. Chest. 2001;119:22S-38S.

57. Schwarz UI, Ritchie MD, Bradford Y, et al. Genetic determinants of response to warfarin during initial anticoagulation. $N$ Engl $J$ Med. 2008;358:999-1008.

58. Pirmohamed M, Burnside G, Eriksson N, et al. A randomized trial of genotype-guided dosing of warfarin. N Engl J Med. 2013;369:2294-2303.

59. Kimmel SE, French B, Kasner SE, et al. A pharmacogenetic versus a clinical algorithm for warfarin dosing. $N$ Engl J Med. 2013;369: 2283-2293.

60. Altman RB. Pharmacogenomics: "noninferiority" is sufficient for initial implementation. Clin Pharmacol Ther. 2011;89:348-350.

61. Ratain MJ, Johnson JA. Meaningful use of pharmacogenetics. Clin Pharmacol Ther. 2014;96:650-652.

62. Frueh FW. Back to the future: why randomized controlled trials cannot be the answer to pharmacogenomics and personalized medicine. Pharmacogenomics. 2009;10:1077-1081.

63. Schwarz UI, Kim RB, Tirona RG. Genotype-guided dosing of vitamin K antagonists. $N$ Engl J Med. 2014;370:1761-1762.

64. Eby C. The pharmacogenetics of vitamin $\mathrm{K}$ antagonist anticoagulation drugs. In: Wu AHB, Yeo K-TJ, eds. Pharmacogenomic Testing in Current Clinical Practice. New York, NY: Springer Science+Business Media; 2011:117-138.

65. Nguyen N, Anley P, Yu MY, et al. Genetic and clinical determinants influencing warfarin dosing in children with heart disease. Pediatr Cardiol. 2013;34:984-990.

66. Kim HS, Lee SS, Oh M, et al. Effect of CYP2C9 and VKORC1 genotypes on early-phase and steady-state warfarin dosing in Korean patients with mechanical heart valve replacement. Pharmacogenet Genom. 2009; 19:103-112.

67. Yang J, Huang C, Shen Z, et al. Contribution of $1173 \mathrm{C}>\mathrm{T}$ polymorphism in the VKORC1 gene to warfarin dose requirements in Han
Chinese patients receiving anticoagulation. Int $J$ Clin Pharm Ther. 2011;49:23-29.

68. Namazi S, Azarpira N, Hendijani F, et al. The impact of genetic polymorphisms and patient characteristics on warfarin dose requirements: a cross-sectional study in Iran. Clin Ther. 2010;32:1050-1060.

69. Ozer N, Cam N, Tangurek B, et al. The impact of CYP2C9 and VKORC1 genetic polymorphism and patient characteristics upon warfarin dose requirements in an adult Turkish population. Heart Vessels. 2010;25:155-162.

70. Palacio L, Falla D, Tobon I, et al. Pharmacogenetic impact of VKORC1 and CYP2C9 allelic variants on warfarin dose requirements in a hispanic population isolate. Clin Appl Thromb Hemost. 2010;16:83-90.

71. Cavallari LH, Momary KM, Patel SR, et al. Pharmacogenomics of warfarin dose requirements in Hispanics. Blood Cells Mol Dis. 2011; 46:147-150.

72. Schelleman H, Chen Z, Kealey C, et al. Warfarin response and vitamin K epoxide reductase complex 1 in African Americans and Caucasians. Clin Pharmacol Ther. 2007;81:742-747.

73. Liang $\mathrm{R}$, $\mathrm{Li} \mathrm{L}$, Li C, et al. Impact of CYP2C9*3, VKORC1-1639, CYP4F2rs2108622 genetic polymorphism and clinical factors on warfarin maintenance dose in Han-Chinese patients. J Thromb Thrombolysis. 2012;34:120-125.

74. Zhang W, Zhang WJ, Zhu J, et al. Genetic polymorphisms are associated with variations in warfarin maintenance dose in Han Chinese patients with venous thromboembolism. Pharmacogenomics. 2012;13:309-321.

75. Ozer M, Demirci Y, Hizel C, et al. Impact of genetic factors (CYP2C9, VKORC1 and CYP4F2) on warfarin dose requirement in the Turkish population. Basic Clin Pharmacol Toxicol. 2013;112:209-214.

76. Shrif NE, Won HH, Lee ST, et al. Evaluation of the effects of VKORC1 polymorphisms and haplotypes, CYP2C9 genotypes, and clinical factors on warfarin response in Sudanese patients. Eur J Clin Pharmacol. 2011; 67:1119-1130. 